\title{
Efficacy of Gynostemma pentaphyllum Extract in Anti-obesity
}

\section{Therapy}

\section{Yoon Hee Kim ${ }^{1}$, So Mi Kim $\odot^{2}$, Jae Kyoung Lee ${ }^{1}$, Sung Kwan Jo ${ }^{1}$, Hyung Joong Kim ${ }^{1}$, Kyu Min Cha ${ }^{1}$, Cho Young Lim ${ }^{1}$, Joo Myung Moon ${ }^{1}$, Tae Young Kim ${ }^{1}$ and Eun Ji Kim ${ }^{2 *}$}

\author{
${ }^{I} R \& D$ Center, BTC Corporation, Ansan 15588, Republic of Korea
}

${ }^{2}$ Center for Efficacy Assessment and Development of Functional Foods and Drugs, Hallym University,

Chuncheon 24252, Republic of Korea

(Received May 22, 2019; Revised August 6, 2019; Accepted August 17, 2019)

\begin{abstract}
In the present study, we investigated a saponin-enriched fraction from Gynostemma pentaphyllum extract (GPE) and isolated gypenoside L (GL), gypenoside LI (GLI), and ginsenoside Rg3 (Rg3). To explore the anti-obesity activities of these compounds, we investigated the effects of GL, GLI, and Rg3 on glucose uptake and activation of AMPK in L6 skeletal muscle cells. GPE, GL, GLI, and Rg3 markedly increased glucose uptake activity and GLUT4 gene expression in L6 myotubes. The enhanced glucose uptake was mediated by the activation of the AMPK-ACC signaling pathway. In addition, we observed that GPE, GL, GLI, and Rg3 increased the expression of SIRT1 and PPAR $\gamma$ coacvtivator-1 $\alpha(P G C-1 \alpha)$. Our results suggest the potential use of Gynostemma pentaphyllum in developing a therapeutic agent to reduce body weight and prevent diet-related diseases via glucose uptake and AMPK activation.
\end{abstract}

Keywords: Gynostemma pentaphyllum; gypenosides; L6 skeletal muscle cells; glucose uptake, AMPK; SIRT1. (C) 2019 ACG Publications. All rights reserved

\section{Introduction}

Obesity, characterized by excessive body fat accumulation, arises from an imbalance between an individuals' energy intake and consumption, causing excess calories to accumulate as lipids in the adipose tissue, muscles, and the liver [1]. Obesity is a risk factor for the development of type 2 diabetes, insulin resistance, hyperlipidemia, hypertension, cardiovascular disease, several types of cancer, and other chronic diseases [2,3]. In order to combat this growing public health problem, consumption of food with active anti-obesity constituents is an important strategy [4].

Gynostemma pentaphyllum (Thunb.) Makino (Cucurbitaceae) has been reported to have antiobesity and anti-diabetic properties, and has been consumed as a tea, in beverages, and in folk medicine for many years. Some pharmaceutical bioactivities found in saponin-rich plants are reported to be enhanced by hydrolysis of saponin glycoside. Hydrolysis occurs during leaves processing methods of raw material such as air-drying, steaming, or parching, and leads to the structural conversion of

\footnotetext{
* Corresponding author: E-mail: myej4@hallym.ac.kr; Phone: (82)-33-248-3106; Fax: (82)-33-244-3107
} 
ginsenosides or gypenosides [5,6], resulting in changes to saponin composition. Previous studies demonstrated that hydrolysis by heat processing changes several saponins of $G$. pentaphyllum to the gypenoside stereoisomers gypenoside L (GL) and gypenoside LI (GLI) [7]. The levels of two dammarane-type saponins were increased by heating and process time when compared to levels in the raw plant. Saponin glucosides (GL and GLI) in G. pentaphyllum are produced from the high-saponin glucosides (gypenoside XLVI and LVI) due to loss of a glycosyl moiety during the heating process [8]. Many studies have aimed to convert major compounds to the more active minor compounds with methods such as heating, acid treatment, or enzymatic conversion. However, the case of acid treatment process could degrade active minor ginsenosides or gypenosides by randomly hydrolyzing all glycosidic bonds. More researches have been focusing on the heating process of minor ginsenoside or gypenosides. Nevertheless, low abundant minor ginsenoside and gypenosides are difficult to obtain because of lower concentration of their natural abundance in raw plants [9].

Earlier investigations of the major phytochemical constituents were established that $G$. pentaphyllum contains dammarane-type glycosides, which are saponins containing one to four saccharides combined with a dammarane backbone [10]. They include protopanaxadiol-type ginsenosides (ginsenosides $\mathrm{Rb} 1, \mathrm{Rg} 3$, and F2) previously isolated from Panax ginseng (Araliaceae) $[11,12]$. The major bioactive constituents of $G$. pentaphyllum are regarded to be gypenosides, which are reported to possess a variety of beneficial biological activities including tumor-suppressive [13], immunomodulatory [14], cholesterol-lowering [15], antioxidant [16], and hypoglycemic effects [17].

GLUT4 is a principal glucose transporter that is stimulated by either insulin or muscle contraction. The primary role of $5^{\prime}$-adenosine monophosphate-activated protein kinase (AMPK) is to regulate this process, taking part in the activation of glucose uptake through GLUT4 translocation, which modifies glucose levels and controls metabolism $[18,19]$. AMPK is a serine/threonine kinase that consists of three subunits: a catalytic $(\alpha)$ and two regulatory $(\beta$ and $\gamma$ ) subunits [18,20-2]. In a situation of diminished intracellular ATP levels, AMPK activates central energy metabolism processes such as fatty acid oxidation, mitochondrial biogenesis, glucose uptake, glycolysis, lipogenesis, and protein synthesis [23,24]. Inactivation of acetyl-CoA carboxylase (ACC) by AMPK results in a decline in malonyl-CoA content. Moreover, inactivation of ACC results in a decrease in fatty acid synthesis and an increase in fatty acid oxidation [1]. AMPK activates NAD-dependent protein deacetylase sirtuin 1 (SIRT-1) and increases peroxisome proliferator activated receptor $\gamma(\operatorname{PPAR} \gamma)$ coactivator-1 $\alpha(P G C-1 \alpha)$ expression in the nucleus. AMPK also affects the downstream activation of mitochondrial biogenesis and modulates the metabolic genes involved in both glucose metabolism and fatty acid oxidation. Previous studies demonstrated that the dammarane-type glucoside gypensapogenin from G. pentaphyllum enhanced 2NBDG uptake and glucose transporter 4 (GLUT4) translocation via activation of the AMPK-ACC signaling pathway [10]. Augmenting the translocation and expression of GLUT4 is a promising therapeutic strategy against obesity.

However, the exact mechanism underlying GLUT4 translocation and the activation of AMPK signaling effects for GL and GLI from G. pentaphyllum remains unclear. The Rg3 in $P$. ginseng has been found to improve insulin signaling and glucose uptake primarily by the expression of insulin receptor substrate-1 (IRS-1) and GLUT4 in L6 myotubes [25] but Rg3 isolated from G. pentaphyllum has not yet been investigated.

Thus, we hypothesized GPE activates AMPK signaling and regulates glucose uptake and metabolic genes expression. Herein, we have studied using a G. pentaphyllum extract (GPE) by a newly patented extraction method for increasing effective saponin compounds. In addition, we identified major compounds GL, GLI and Rg3, three saponins isolated from GPE (Supporting information Figure S1). We then examined a search for bioactive gypenosides with anti-obesity effects, phytochemically investigated GPE, GL, GLI, and Rg3.

\section{Materials and Methods}

\subsection{Materials and Reagents}

The materials used in this study were purchased from the indicated suppliers: High-performance liquid chromatography (HPLC)-grade acetonitrile and metformin from Sigma-Aldrich Co. (St. Louis, 
Anti-obesity effects of Gynostemma pentaphyllum extract

MO, USA); Dulbecco's modified Eagle's medium (DMEM) and other cell culture reagents from Welgene (Daegu, Korea); Glucose uptake assay kit from Biovision Inc. (Milpitas, CA, USA); Antibodies against AMPK, p-AMPK, ACC, p-ACC, and $\beta$-actin from Cell Signaling Technology (Beverly, MA, USA); GLUT4 antibody from Santa Cruz Biotechnology Inc. (Santa Cruz, CA, USA); BCA protein assay kit and Trizol reagent from Thermo Scientific (Rochford, IL, USA); Immobilon ${ }^{\mathrm{TM}}$ Western Chemiluminescent HRP Substrate from Millipore Corporation (Billerica, MA, USA); RotorGene $^{\mathrm{TM}}$ SYBR Green kit from Qiagen (Hilden, Germany).

\subsection{Isolation and Purification of GL, GLI, and Rg3 from GPE}

GPE (Lot number. BGPL170518) and highly purified (> 98\%) saponin standards GL, GLI, and Rg3 were prepared by BTC Corporation (Ansan, Korea). Leaves of G. pentaphyllum were parched using an electric-heat-controller and dried. Extracts were prepared from the leaves of G. pentaphyllum $(10 \mathrm{~kg})$ with hot-water $(20 \mathrm{~L})$ and $50 \% \mathrm{EtOH}(15 \mathrm{~L})$ aqueous solution using a newly patented method (Patent No: KR101969062; "Preparation method of Gynostemma Pentaphyllum leaves extract for increasing small molecular effective saponin contents, and decreasing benzopyrene, and Gynostemma pentaphyllum extract using thereof"). The two resultant supernatants were collected and combined, and then filtered. The filtrate was vacuum-evaporated to obtain GPE. Quantification of GL, GLI, and Rg3 was performed by high-performance liquid chromatography (HPLC) analysis. GPE was dissolved in 3\% dimethylsulfoxide and used for the bioactivity assays.

GPE powder $(100 \mathrm{~g})$ was suspended in $\mathrm{H}_{2} \mathrm{O}(1 \mathrm{~L})$ and successively partitioned with $\mathrm{n}$-hexane $(1 \mathrm{~L}$ $\times 3)$, EtOAc $(1 \mathrm{~L} \times 3)$, and $\mathrm{BuOH}(1 \mathrm{~L} \times 3)$. The $\mathrm{BuOH}$ fraction was subjected to HP-20 ion exchange resin column chromatography $(50 \times 20 \mathrm{~cm})$ and eluted by sequential elution by increasing $\mathrm{MeOH}$ levels in $\mathrm{H}_{2} \mathrm{O}$. The $\mathrm{MeOH}$ fraction was obtained and evaporated, then passed through a silica gel column F60 $(65 \times 15 \mathrm{~cm})$ with a gradient solvent system of $\mathrm{CH}_{2} \mathrm{Cl}_{2}-\mathrm{MeOH}-\mathrm{H}_{2} \mathrm{O}$ (from 5:1:0 to 0:5:0.1 v/v/v) to yield seven sub-fractions (GPE $11 \sim$ GPE 20). Finally, sub-fraction GPE 15 was subjected to a semipreparative HPLC column (SHIM-pack PREP-ODS(L) $250 \times 50 \mathrm{~mm}$ ) and eluted with $47 \%$ acetonitrile in water at a flow rate of $5 \mathrm{~mL} / \mathrm{min}$ to produce compounds 1,2 , and 3 . The structures of the compounds were characterized by ${ }^{1} \mathrm{H}-\mathrm{NMR},{ }^{13} \mathrm{C}-\mathrm{NMR}$, and LC-MS as GL, GLI, and Rg3 spectra (S1). NMR data were consistent with that of the reference $[5,26]$, and the compounds were eventually identified as GL, GLI, and Rg3 (Figure 1). The detailed spectral data are provided in the Supporting Information of this article.

\subsection{Cell Culture and Treatment}

L6 skeletal muscle cells were purchased from the Korean Cell Line Bank (Seoul, Korea). L6 cells were cultured in DMEM containing $100 \mathrm{~mL} / \mathrm{L}$ Fetal Bovine Serum (FBS) with 100,000 U/L penicillin and $100 \mathrm{mg} / \mathrm{L}$ streptomycin at $37{ }^{\circ} \mathrm{C}$ in a humidified atmosphere comprising $5 \% \mathrm{CO}_{2}$ and $95 \%$ air. Myotube differentiation was induced by utilizing L6 cells that were incubated in DMEM supplemented with $20 \mathrm{~mL} / \mathrm{L}$ FBS for four days. The effects of GPE, GL, GLI, and Rg3 on AMPK activation were determined with cells plated in $100 \mathrm{~mm}$ dishes at $1 \times 10^{5}$ cells/dish in DMEM containing $100 \mathrm{~mL} / \mathrm{L}$ FBS. Twenty-four hours after plating, the cells were either treated with the indicated concentrations of GPE, GL, GLI, or Rg3 for $2 \mathrm{~h}$, or metformin (positive control) for $1 \mathrm{~h}$. The effects of GPE, GL, GLI, and $\operatorname{Rg} 3$ on GLUT4 expression and translocation were determined with cells plated in $100 \mathrm{~mm}$ dishes at $1 \times 10^{5}$ cells/dish in DMEM containing $100 \mathrm{~mL} / \mathrm{L}$ FBS. Twenty-four hours after this plating, cells were serum-deprived with DMEM and supplemented with $20 \mathrm{~mL} / \mathrm{L}$ FBS for 4 days to induce myotube differentiation. Following differentiation induction, cells were treated with the indicated concentrations of GL, GLI, Rg3, GPE, or metformin for $4 \mathrm{~h}$. Cells were then harvested and lysed for all experiments.

\subsection{Measurement of Glucose Uptake}

L6 cells were seeded into 96-well culture plates at a density of $1 \times 10^{4}$ cells/well and incubated for $24 \mathrm{~h}$. The cells were then cultured in DMEM containing $20 \mathrm{~mL} / \mathrm{L}$ FBS for 4 days to determine further differentiation. Next, the cells were treated with GL, GLI, Rg3, GPE (2 h), $100 \mathrm{nM}$ insulin $(1 \mathrm{~h})$, or 
vehicle control. Glucose uptake was estimated using a glucose uptake colorimetric assay kit (Biovision) according to the manufacturer's instructions. Independent experiments were conducted, comparing the control group (Con), insulin group (I), and added treatment groups (GPE, GL, GLI, and Rg3).

\subsection{Western Blotting}

Cells were treated with the indicated concentration of GPE, GL, GLI, Rg3, or metformin as described above. The cells were then lysed using the PRO-PREP ${ }^{\mathrm{TM}}$ protein extraction solution (iNtRON Biotechnology, Inc., Seongnam, Korea) according to the manufacturer's instructions. The protein contents of the cell lysates were measured using a BCA protein assay kit (Thermo Scientific, Rochford, IL, USA). Equal amounts of protein were separated by $10 \%(\mathrm{v} / \mathrm{v})$ SDS-PAGE and transferred onto PVDF membranes. These membranes were then incubated overnight at $4{ }^{\circ} \mathrm{C}$ with primary antibodies for GLUT4, IR, p-AMPK (Thr172), AMPK $\alpha$, p-ACC (Ser79), ACC, or $\beta$-actin and were then continually incubated with corresponding horseradish peroxidase-conjugated secondary antibodies for $2 \mathrm{~h}$. Immunoreactive bands were visualized using Immobilon ${ }^{\mathrm{TM}}$ Western Chemiluminescent HRP Substrate (Millipore). The intensity of the protein bands was analyzed using an Image Quant LAS 500 imaging system (GE Healthcare Bio-Sciences AB, Uppsala, Sweden).

\subsection{Real-time RT-PCR}

Expression of SIRT1 and PGC-1 $\alpha$ were analyzed using Real time PCR. Total RNA was extracted using Trizol Reagent (Thermo Fisher Scientific). The content and purity of the total RNA were estimated using a micro-volume spectrophotometer (BioSpec-nano, Shimadzu, Kyoto, Japan). Total RNA was reverse-transcribed to single-strand cDNA using a $1^{\text {st }}$ strand cDNA kit (Tech \& Innovation, Chuncheon, Korea). All quantitative real-time PCR reaction were performed using a Rotor-gene 3000 PCR machine (Corbett Research, Mortlake, NSW, Australia) and the amplifications were done using the Rotor-Gene ${ }^{\mathrm{TM}}$ SYBR Green kit (Qiagen). The sequences of the primers used in this study are shown in Table 1. The PCR amplification of thermal cycling conditions were composed of $94{ }^{\circ} \mathrm{C}$ for $3 \mathrm{~min}, 40$ cycles at $95{ }^{\circ} \mathrm{C}$ for $10 \mathrm{~s}, 60{ }^{\circ} \mathrm{C}$ for $15 \mathrm{~s}$, and $72{ }^{\circ} \mathrm{C}$ for $20 \mathrm{~s}$. Results were analyzed with a Rotor-Gene 6000 Series system software program, version 6 (Corbett Research). The expression levels of genes were normalized to those of glyceraldehyde 3-phosphate dehydrogenase (GAPDH).

Table 1. Gene-specific primers used for real-time PCR analysis

\begin{tabular}{cll}
\hline Primer & Type & \multicolumn{1}{c}{ Sequence $\left(5^{\prime}-3^{\prime}\right)$} \\
\hline \multirow{2}{*}{ SIRT1 } & Forward & CAGTGTCATGGTTCCTTTGC \\
& Reverse & CACCGAGGAACTACCTGAT \\
& Forward & ATCTACTGCCTGGGGACCTT \\
PGC-1 $\alpha$ & Reverse & ATGTGTCGCCTTCTTGCTCT \\
& Forward & CATCAAGAAGGTGGTGAAGCAGG \\
GAPDH & Reverse & CCACCACCCTGTTGCTGTAGCCA \\
\hline
\end{tabular}

\subsection{Statistical Analysis}

Data were presented as mean \pm standard error of mean (SEM) of at least three independent experiments. All statistical analyses were performed using SPSS and GraphPad Prism 4.0 (GraphPad Software Inc., La Jolla, CA, USA). Differences between groups were considered significant at $p<0.05$ by Student's t-test or one-way analysis of variance (ANOVA). 
Anti-obesity effects of Gynostemma pentaphyllum extract

\section{Results and Discussion}

\subsection{Identification of Compounds Isolated from GPE}

GPE was isolated using a novel patented extraction method, which increased the concentration of GL, GLI, and Rg3. This newly developed method was subsequently applied to the simultaneous determination of three major compounds and included GPE:GL [26], GLI [26], and Rg3 [5]. Based upon reported MS and NMR data as well as other physical properties, these compounds were successfully isolated from GPE with purities above $98 \%$. The detailed spectral data are provided in the Supporting Information of this article. The known structures of GL, GLI, and Rg3 are shown in Figure 1.

These three compounds have similar molecular formulas with minor differences in the glucose position. Concentrations of GL, GLI, and Rg3 found in the GPE used in this study were measured by HPLC analysis. Table 2 indicates that $1.8 \pm 0.011 \%$ of GL, $1.4 \pm 0.009 \%$ of GLI, and $0.14 \pm 0.003 \%$ of Rg3 were present in GPE. Based on these values and the molecular weights of GL (MW: $801.01 \mathrm{~g} / \mathrm{mol}$ ), GLI (MW: $801.01 \mathrm{~g} / \mathrm{mol}$ ), and Rg3 (MW: $785.025 \mathrm{~g} / \mathrm{mol}$ ), we calculated the corresponding concentrations and found $0.449 \mu \mathrm{mol} / \mathrm{L}$ GL, $0.35 \mu \mathrm{mol} / \mathrm{L} \mathrm{GLI}$, and $0.038 \mu \mathrm{mol} / \mathrm{L} \mathrm{Rg} 3$ in the media containing $20 \mu \mathrm{g} / \mathrm{mL}$ of GPE (Table 2). However, the concentrations of GL, GLI, and Rg3 in GPE might depend on extraction and storage methods. High concentrations of the GL, GLI, and Rg3 treatments were used to evaluate dose-response and efficiency based on the cell viability results. An MTT assay showed no cytotoxic effects from the ingredients of the GPE or its ingredients in differentiated L6 cells at different concentrations (data not shown).

Table 2. Concentrations of effective components GL, GLI, Rg3 in GPE from Gynostemma pentaphyllum.

\begin{tabular}{ccccccc}
\hline Compound & $\begin{array}{c}\text { Concentration } \\
(\mu \mathrm{g} / \mathrm{mg} \text { in GPE })\end{array}$ & $\begin{array}{c}\text { Concentration in } \\
\begin{array}{c}\mu \mathrm{g} / \mathrm{mL} \text { of GPE } \\
(\mu \mathrm{mol} / \mathrm{L})\end{array}\end{array}$ & $\begin{array}{c}\text { Linear } \\
\text { regression }\end{array}$ & $\mathrm{r}^{\mathrm{a}}$ & LOD $^{\mathrm{b}}$ & $\mathrm{LOQ}^{\mathrm{c}}$ \\
\hline $\mathbf{1}, \mathrm{GL}$ & $1.800 \pm 0.011$ & $0.449 \pm 0.002$ & $\mathrm{y}=3.373 \mathrm{x}-0.183$ & 0.999 & 0.726 & 2.201 \\
$\mathbf{2}, \mathrm{GLI}$ & $1.400 \pm 0.009$ & $0.350 \pm 0.002$ & $\mathrm{y}=3.150 \mathrm{x}-7.523$ & 0.999 & 0.941 & 2.850 \\
$\mathbf{3}, \mathrm{Rg} 3$ & $0.150 \pm 0.003$ & $0.038 \pm 0.001$ & $\mathrm{y}=3.134 \mathrm{x}-0.422$ & 0.999 & 1.130 & 3.430 \\
\hline
\end{tabular}

${ }^{\mathrm{a}}$ Correlation coefficients of the regression equation. ${ }^{\mathrm{b}}$ LOD $=$ limit of detection. ${ }^{\mathrm{c}}$ LOQ $=$ limit of quantification.

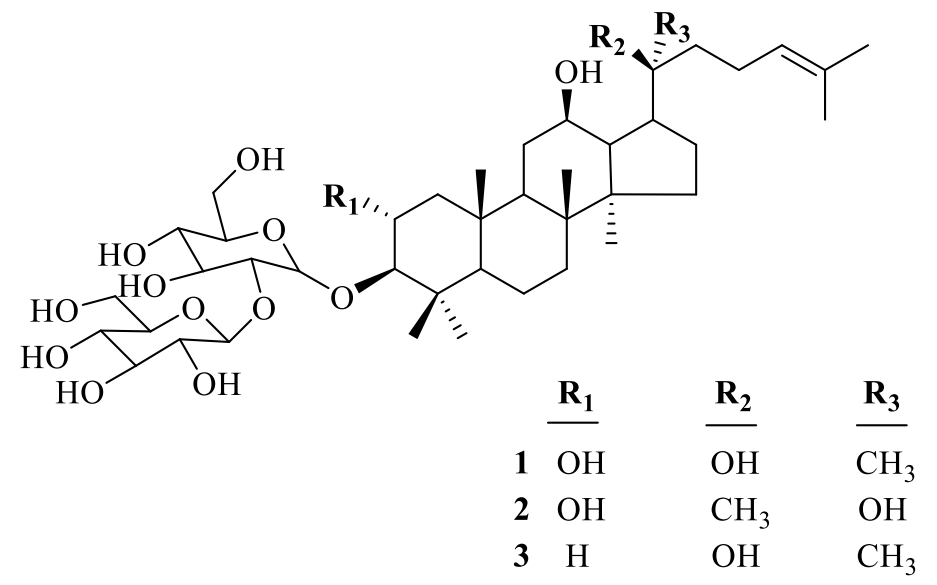

Figure 1. Chemical structures of compound $\mathbf{1}(\mathrm{GL}), \mathbf{2}(\mathrm{GLI})$, and $\mathbf{3}(\mathrm{Rg} 3)$ isolated from Gynostemma pentaphyllum 


\subsection{Stimulation of Glucose Uptake in L6 Muscle Cells}

We next aimed to assess the effects of each of the gypenosides found in GPE (GL, GLI, and Rg3) on the efficiency of glucose uptake. This efficiency was determined via evaluation of the effects of GPE on the glucose uptake in cultured muscle cells, L6 cells were then differentiated into myotubes and incubated with $100 \mathrm{nM}$ insulin or different concentrations of GL, GLI, Rg3, or GPE for $2 \mathrm{~h}$. GPE administration resulted in a dose-dependent increase in glucose uptake. Compared with that of the control group, a significantly increased response was observed with 20 and $50 \mu \mathrm{g} / \mathrm{mL}$ of GPE. Importantly, the response of these cells to $50 \mu \mathrm{g} / \mathrm{mL}$ of GPE was higher than that of $100 \mathrm{nM}$ insulin. The effects of GL, GLI, and Rg3 on glucose uptake in the L6 cells was investigated. GL and GLI were found to markedly increase glucose uptake in L6 cells (Figure 2). Notably, the response of these cells to $20 \mu \mathrm{M}$ GL was comparable to that to $50 \mu \mathrm{M}$ GL. Further, exposure to different concentrations of GLI resulted in a dose-dependent increase in glucose uptake. Moreover, the responses to 20 and $50 \mu \mathrm{M} \mathrm{Rg} 3$ were increased compared to those to $100 \mathrm{nM}$ insulin. These results indicated that GL, GLI, Rg3, and GPE all increased glucose uptake activity in L6 myotubes.

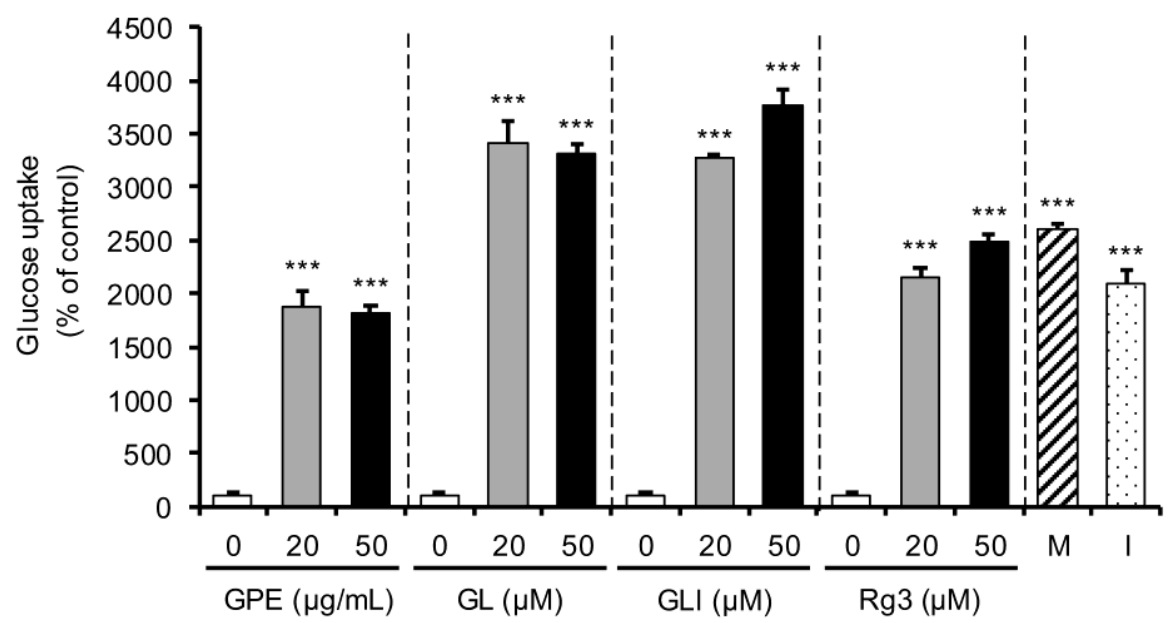

Figure 2. Effect of GPE, GL, GLI, and Rg3 on glucose uptake in L6 cells.

The differentiated L6 cells were treated with the indicated concentrations of GPE, GL, GLI, and Rg3, $100 \mathrm{nM}$ insulin (I), and $2 \mathrm{mM}$ metformin (M). Values are expressed as mean \pm SEM $(\mathrm{n}=4) .{ }^{*} P<0.05,{ }^{* * *} P<0.01,{ }^{* * * *} P<$ 0.01 indicate a significant difference from non-treated cells (control)

\subsection{Increased Expression of GLUT4 in L6 Cells}

We then attempted to determine the effect of the gypenosides from GPE on GLUT4 expression via western blot analysis. GLUT4 protein expression significantly increased in a dose-dependent manner following treatment of the L6 cells with different concentrations of GPE, GL, GLI, or Rg3 (Figure 3A and $3 \mathrm{~B}$ ). These results suggested that GLUT4 translocation may be responsible for GPE-induced glucose uptake. GPE and its ingredients increased GLUT4 translocation to the plasma membrane, and also promoted the expression of GLUT4 in L6 cells. 
Anti-obesity effects of Gynostemma pentaphyllum extract
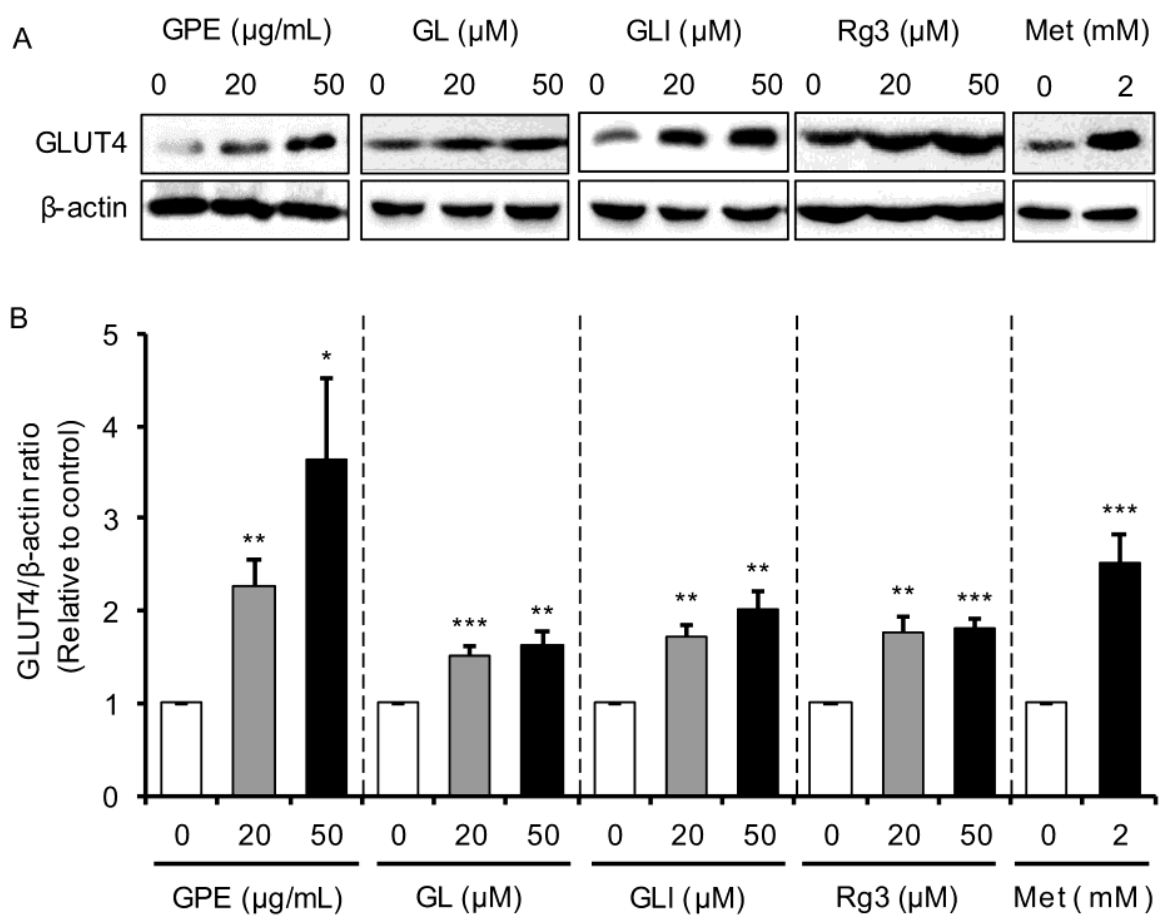

Figure 3. Effect of GPE, GL, GLI, and Rg3 on GLUT4 expression in L6 cells

The differentiated L6 cells were treated with the indicated concentration of GPE, GL, GLI, Rg3, and metformin (Met). The expression of GLUT4 in cell was analyzed via Western blotting with the indicated antibodies. (A) Photographs of chemiluminescent detection of the blots, which are representative of three independent experiments, are shown. (B) The relative abundance of each band to its own $\beta$-actin was quantified, and the control levels were set at 1 . Values are expressed as the adjusted mean $\pm \operatorname{SEM}(\mathrm{n}=3) .{ }^{*} P<0.05,{ }^{* *} P<0.01,{ }^{* * *} P$ $<0.01$ indicate a significant difference from non-treated cells (control).

\subsection{Promotion of AMPK Expression and ACC Phosphorylation/Activation in L6 Cells}

GLUT4 is triggered through the AMPK signaling pathway and could be activated by anti-diabetic compounds. The mechanism underlying GPE-induced glucose uptake was determined via the observed effects of GL, GLI, Rg3, GPE, and metformin on AMPK phosphorylation/activation. Treatment with GPE resulted in an increase in the AMPK phosphorylation ratio that was comparable to the effect of metformin (Figure 4A and B). AMPK phosphorylation significantly increased in a dose-dependent manner after treatment with GL and Rg3. However, no significant activity was observed in response to $20 \mu \mathrm{g} / \mathrm{mL}$ GPE.

In addition, we examined phosphorylation of ACC, downstream effector of AMPK. AMPK phosphorylated ACC at Ser-79 and inhibited further activity. ACC catabolized the carboxylation of acetyl CoA to malonyl-CoA, which played a key role in fatty acid synthesis and $\beta$-oxidation of long chain fatty acids. GL, GLI, and $\operatorname{Rg} 3$ all increased the phosphorylation ratio of ACC. GL and GLI showed significant effects; while, Rg3 showed no significant difference. Treatment with GPE resulted in a significant increase in the phosphorylation of ACC, which was higher than that induced by metformin, or the GPE ingredients individually (Figure 4A and C). Thus, GL, GLI, and Rg3 might have acted synergistically in the activation of ACC. 

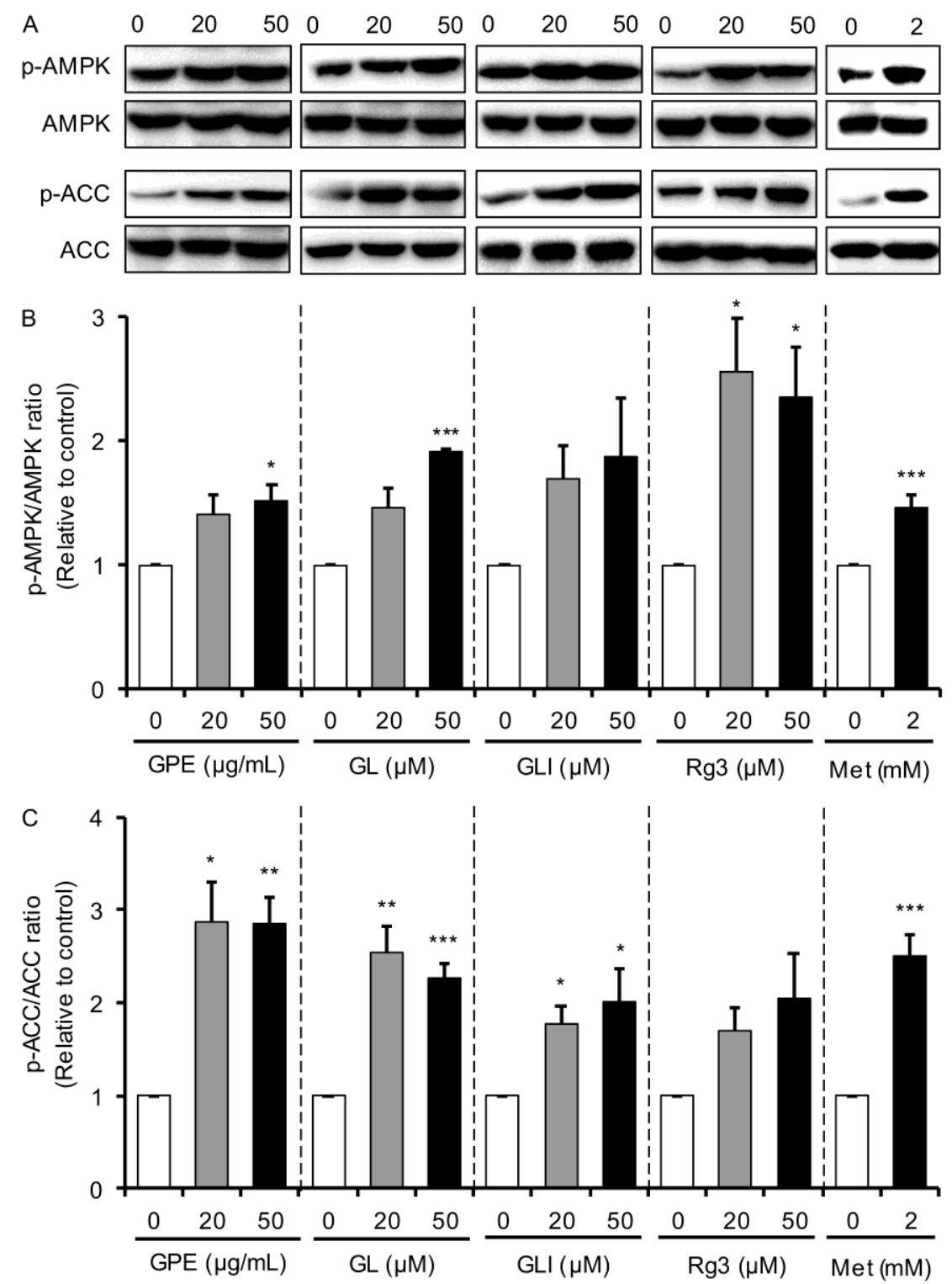

Figure 4. Effect of GPE, GL, GLI, and Rg3 on AMPK and ACC phosphorylation in L6 cells L6 cells were treated with the indicated concentrations of GPE, GL, GLI, Rg3, and metformin (Met). The phosphorylation of AMPK and ACC were analyzed via western blotting with the indicated antibodies. (A) Photographs of chemiluminescent detection of the blots, which are representative of three independent experiments, are shown. (B) The relative fold change in the intensity of the phosphorylated protein to total protein bands was quantified, and the control levels were set at 1 . Values are expressed as the adjusted mean \pm SEM $(\mathrm{n}=$ 3). ${ }^{*} P<0.05,{ }^{* *} P<0.01,{ }^{* * *} P<0.01$ indicate a significant difference from non-treated cells (control).

\subsection{Increased AMPK-dependent Gene Expression}

In order to determine whether GPE influenced lipid and glucose metabolism through AMPKdependent gene expression, mRNA expression of SIRT1 and PGC-1 $\alpha$ was investigated with real-time PCR. The expression level of SIRT1 following treatment with $20 \mu \mathrm{g} / \mathrm{mL}$ GPE was similar to that in cells treated with metformin and higher than that by treatment with $50 \mu \mathrm{g} / \mathrm{mL}$ GPE (Figure 5). On the other hand, the compounds GL, GLI, and Rg3 dose-dependently increased mRNA levels of SIRT1 in L6 cells. However, no significant activity was observed in response to the GL treatment. These results suggested that GL, GLI, Rg3, and GPE regulate glucose homeostasis and energy metabolism via enhanced SIRTI expression. $P G C-1 \alpha$, which is a major regulator of mitochondrial biogenesis was also evaluated. Our 
results found significant increases in the activation of $P G C$ - $1 \alpha$ following treatment with GL, GLI, Rg3, and GPE. Moreover, expression of $P G C$ - $1 \alpha$ was increased following treatment with GPE at a lower dose, by approximately 4.95 -fold compared to that of the control.
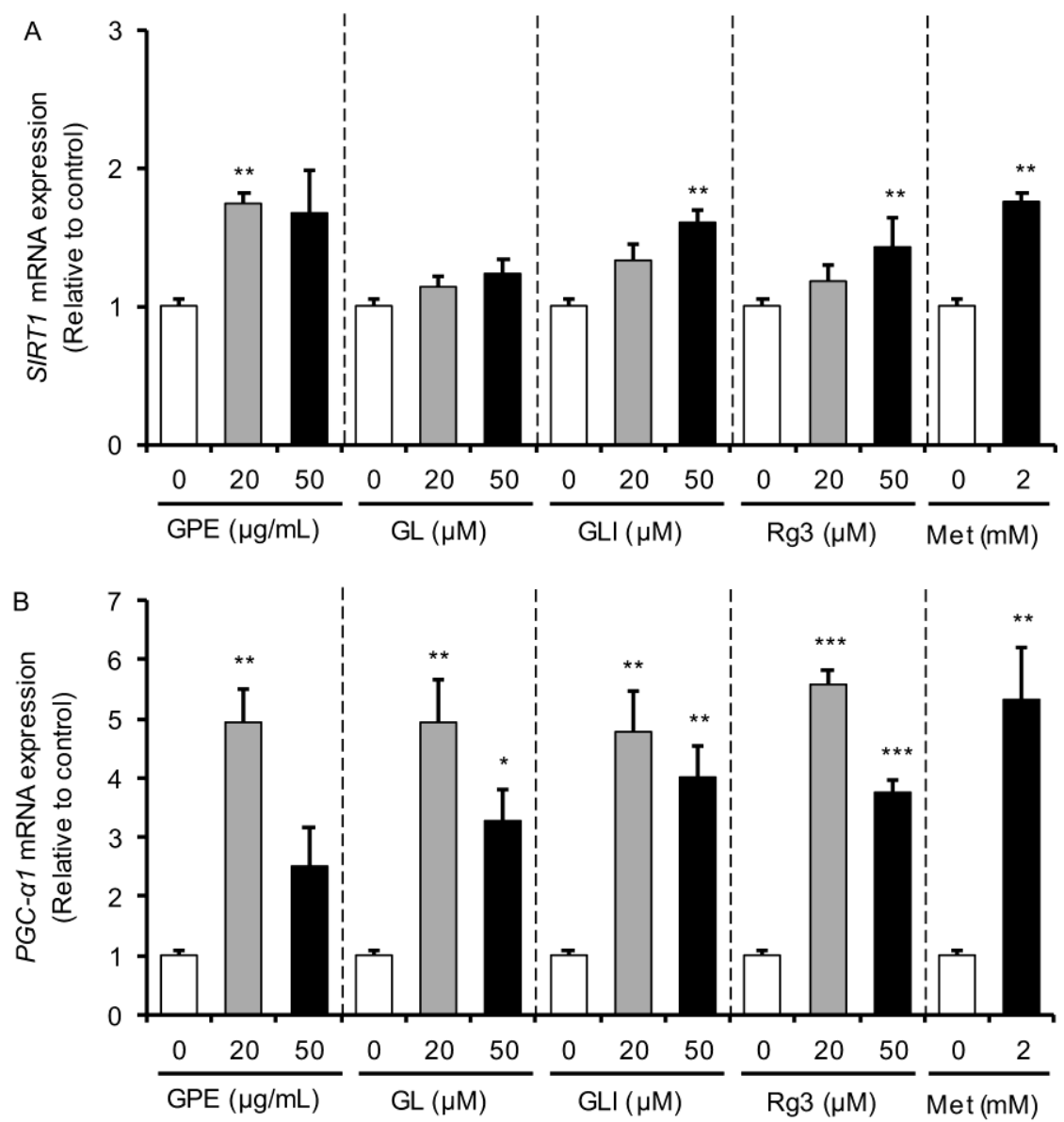

Figure 5. Effect of GPE, GL, GLI, and Rg3 on SIRT1 and PGC- $\alpha 1$ mRNA expression in L6 cells L6 cells were treated with the indicated concentrations of GPE, GL, GLI, Rg3, and metformin (Met). Total RNA was isolated and real-time RT-PCR for SIRTI and $P G C$ - $\alpha l$ was performed. Values are expressed as mean \pm SEM $(\mathrm{n}=3) .{ }^{*} P<0.05,{ }^{* *} P<0.01,{ }^{* * *} P<0.01$ indicate a significant difference from non-treated cells (control).

In recent decades, obesity has become a serious global public health concern. Increased body fat is due to an imbalance between energy intake and consumption. It is also associated with various diseases including hyperlipidemia, diabetes, and fatty liver. However, the use of anti-obesity drugs such as orlistat and sibutramine are reported to cause serious side effects. Consequently, the use of herbal medicines to treat obesity has become the subject of increased attention due to their natural origin, cost effectiveness, and minimal side effects [27, 28]. Low bioavailability has been a concern, because it can limit or even hinder the therapeutic effectiveness of herbal remedies. Thus, poor bioavailability remains the major challenge in developing clinically useful functional food and medicines. A successful therapy requires an appropriate extraction method yielding high contents of effective compounds that could enhance their bioavailability and offer greater efficacy.

G. pentaphyllum, known as "Southern ginseng" is a popular herbal medicine that has traditionally been used throughout centuries for its nutritional and medicinal properties. A research study by Yun et al. has demonstrated that gypenosides could be utilized to promote weight loss as it regulated fat metabolism without causing side effects, such as diarrhea or altered appetite [29]. Other studies have found that G. pentaphyllum extract acted against various diseases and possessed hypoglycemic [13], immunomodulating [14], cholesterol-lowering [15], antioxidant [16], and other activities. The dammarane saponins isolated from G. pentaphyllum, specifically gypenosides, have served as 
economically important raw materials for the food and pharmaceutical industries. We obtained GPE by our newly patented extraction technique, which increased the concentrations of GL, GLI, and Rg3. This extraction process was developed to increase the yield of bioactive compounds from G. pentaphyllum. Following the process, we found that GPE consists of $1.8 \pm 0.011 \%$ GL, $1.4 \pm 0.009 \%$ GLI, and $0.14 \pm$ $0.003 \% \operatorname{Rg} 3$. These compounds usually contain much lower proportions in the raw plants.

Glucose uptake into the peripheral adipose tissues and skeletal muscle required insulin, which stimulated fat and muscle cells to take up excess circulating glucose. Thus, the insulin stimulated translocation of GLUT4 storage vesicles (GSV) from the intracellular storage pools to the plasma membrane (PM). Upon deposition of the GLUT4 protein on the PM, glucose uptake was increased by 10- to 20-fold [30-32]. Thus, enhancing translocation and expression of GLUT4 might be a promising anti-obesity strategy. Zhao et al.[33] reported that dandelion chloroform extract (DCE) induced increased glucose uptake by L6 cells, and also significantly enhanced insulin-responsive aminopeptidase (IRAP) and GLUT4 expression of the cell membrane. These data suggest that DCE promoted GLUT4 translocation to the plasma membrane. Oh and Yang et al. reported that triterpenes from $G$. pentaphyllum significantly stimulated GLUT4 translocation to the plasma membrane, but also upregulated phosphorylation of AMPK and ACC in 3T3-L1 adipocytes [6]. Other dammarane-type triterpenes isolated from Gynostemma longipes, which included secolongipegenins S1 and S2, were reported to increase glucose uptake and GLUT4 translocation to the cytoplasmic membrane within L6 myotube cells [34]. In the present study, treatment of the cells with GL, GLI, Rg3, and GPE stimulated glucose uptake and GLUT4 translocation within the plasma membrane of the L6 myotubes (Figure 2 and 3). On the other hand, the effects of the anti-diabetic drug metformin on muscle glucose uptake were more prolonged than those of insulin. In the present study (based on the data obtained from other studies) we exposed cells to metformin for a minimum of $1 \mathrm{~h}$ as a control [38]. Moreover, previous studies have linked insulin and metformin, and our data supported this; thereby, it was assumed that the increase in glucose uptake observed following treatments was due to GLUT translocation. We therefore measured GLUT4 levels in cell lysates and documented the increased responses to treatment. These results further supported the hypothesis that GL, GLI, Rg3, and GPE impart beneficial effects to both glucose uptake and energy metabolism.

AMPK is an energy regulator and metabolic switch that phosphorylates key targeted proteins involved in lipid metabolism, fatty acid oxidation, and glucose uptake. The underlying mechanism for stimulation of glucose uptake was evaluated by the effects of GPE and isolated compounds on AMPK activation. The effect of GPE and the other isolated compounds on the phosphorylation of AMPK and ACC was significantly increased by utilizing differentiated L6 cells. These results suggested that the isolated compounds GL, GLI, Rg3, and GPE enhanced glucose uptake by regulating the AMPK signaling pathway. Several studies have reported changes in mitochondrial function and mass, damaged oxidative capacity, and altered expression of $P G C-1 \alpha$ in the skeletal muscle of insulin-resistant individuals. Transcription factors involved in this pathway included nuclear respiratory factors (NRF1/NRF-2), mitochondrial transcription factor A (Tfam), and PGC-1 $\alpha$. An accompanying increase in mitochondrial biogenesis involved activation of calcium/calmodulin-dependent protein kinase (CaMK), p38 mitogen-activated protein kinase [35, 36], AMPK [37], as well as phosphorylation of $P G C-1 \alpha$ at Thr177 and Ser538. The activity of PGC-1 $\alpha$ was influenced by AMPK [38], p38 MAPK [35, 36], and protein kinase B (PKB) [39], which modified stability and activity [40]. PGC-1 $\alpha$ deacetylation has already been demonstrated to occur via SIRT1 in vitro as well as in vivo [41, 41]. In addition, the interactions between the deacetylation of $P G C$-1 $\alpha$ by SIRTI and AMPK have been investigated in detail by other research [42]. Furthermore, exercise is involved with increased $P G C-1 \alpha$ expression in the muscle, heart, and adipose tissues [36, 43]. Thus, endurance training might determine whether the CaMK or AMPK pathway is primarily activated [44]. In the present study, treatment with GL, GLI, Rg3, and GPE stimulated mRNA expression of SIRT1 and PGC-1 $\alpha$. Further, the effective gypenosides (GL, GLI, and Rg3) in conjunction with GPE significantly enhanced glucose uptake and GLUT4 levels and activated AMPK. This study thereby indicated the potential of GL, GLI, Rg3, and GPE as candidates for use as an anti-obesity treatment.

Overall, our study shows that GPE from G. pentaphyllum, and its three isolated compounds GL, GLI, and $\operatorname{Rg} 3$, stimulate glucose uptake via a mechanism involved with the AMPK signaling pathway within L6 myotubes. Our results provide new information related to the anti-obesity activities of GPE, 
Anti-obesity effects of Gynostemma pentaphyllum extract

GL, GLI, and Rg3, and highlight their potential uses as therapeutic agents for mediating metabolic disorders. Finally, we suggest that our results justify the use of a diet-induced animal model of obesity to further define the therapeutic role of GPE, GL, GLI, and Rg3 in vivo.

Acknowledgments

We would like to thank the BTC Corporation for providing the GPE.

\section{Author Disclosure Statement}

No competing financial interests exist.

\section{Conflicts of Interest}

The authors declare no conflicts of interest regarding the publication of this paper.

\section{Supporting Information}

Supporting information accompanies this paper on http://www.acgpubs.org/journal/records-ofnatural-products

\section{ORCID}

Yoon Hee Kim: 0000-0002-6045-9927

So Mi Kim: 0000-0002-0174-3485

Jae Kyoung Lee: 0000-0002-5296-4560

Sung Kwan Jo: 0000-0002-2446-3099

Hyung Joong Kim: 0000-0003-1101-7388

Kyu Min Cha: 0000-0002-8864-7962

Cho Young Lim: 0000-0002-2171-6847

Joo Myung Moon: 0000-0002-5750-9620

Tae Young Kim: 0000-0003-1428-1176

Eun Ji Kim: 0000-0001-9305-4769

\section{References}

[1] B. Viollet and F. Andreelli (2011). AMP-activated protein kinase and metabolic control, Handb. Exp. Pharmacol. 203, 303-330.

[2] Y.K. Park, J. Lee, V.S. Hong, J.S. Choi, T.Y. Lee and B.C. Jang (2014). Identification of KMU-3, a novel derivative of gallic acid, as an inhibitor of adipogenesis, PLoS One 9, e109344.

[3] J.H. Lee, M.H. Moon, J.K. Jeong, Y.G. Park, Y.J. Lee, J.W. Seol and S.Y. Park (2012). Sulforaphane induced adipolysis via hormone sensitive lipase activation, regulated by AMPK signaling pathway, Biochem. Biophys. Res. Commun. 426, 492-497.

[4] J.T. Hwang, M.S. Lee, H.J. Kim, M.J. Sung, H.Y. Kim, M.S. Kim and D.Y. Kwon (2009). Antiobesity effect of ginsenoside Rg3 involves the AMPK and PPAR-gamma signal pathways, Phytother. Res. 23, 262-266.

[5] F. Soldati and O. Tanaka (1984). Panax ginseng: Relation between age of plant and content of ginsenosides, Planta Med. 50, 351-352.

[6] J. Wang, T.K.Q. Ha, Y.P. Shi, W.K. Oh and J.L. Yang (2018). Hypoglycemic triterpenes from Gynostemma pentaphyllum, Phytochemistry 155, 171-181.

[7] Y. Jin, Y.J. Kim, J.N. Jeon, C. Wang, J.W. Min, H.Y. Noh and D.C. Yang (2015). Effect of white, red and black ginseng on physicochemical properties and ginsenosides, Plant Foods Hum. Nutr. 70, 141-145.

[8] S.B. Lim (2017). Enhancement of biological activity of saponin-rich plants by subcritical water hydrolysis, Int. J. Clin. Nutr. Diet. 3, 117 (4 pages). 
[9] L.Q. Cheng, J.R. Na, M.K. Kim, M.H. Bang and D.C. Yang (2007). Microbial conversion of ginsenoside $\mathrm{Rb} 1$ to minor ginsenoside F2 and gypenoside XVII by Intrasporangium sp. GS603 isolated from soil, J. Microbiol. Biotechnol. 17, 1937-1943.

[10] K.S. Kang, J. Ham, Y.J. Kim, J.H. Park, E.J. Cho and N. Yamabe (2013). Heat-processed Panax ginseng and diabetic renal damage: active components and action mechanism, J. Ginseng Res. 37, 379-388.

[11] T.H. Huang, V. Razmovski-Naumovski, N.K. Salam, R.K. Duke, V.H. Tran, C.C. Duke and B.D. Roufogalis (2005). A novel LXR-alpha activator identified from the natural product Gynostemma pentaphyllum, Biochem. Pharmacol. 70, 1298-1308.

[12] L. Hu, Z. Chen and Y. Xie (1996). New triterpenoid saponins from Gynostemma pentaphyllum, J. Nat. Prod. 59, 1143-1145.

[13] K.W. Lu, Y.S. Ma, F.S. Yu, Y.P. Huang, Y.L. Chu, R.S. Wu, C.L. Liao, F.S. Chueh and J.G. Chung (2017). Gypenosides induce cell death and alter gene expression in human oral cancer HSC-3 cells, Exp. Ther. Med. 14, 2469-2476.

[14] W.C. Huang, M.L. Kuo, M.L. Li, R.C. Yang, C.J. Liou and J.J. Shen (2008). Gynostemma pentaphyllum decreases allergic reactions in a murine asthmatic model, Am. J. Chin. Med. 36, 579-592.

[15] Norberg, N.K. Hoa, E. Liepinsh, D. Van Phan, N.D. Thuan, H. Jornvall, R. Sillard and C.G. Ostenson (2004). A novel insulin-releasing substance, phanoside, from the plant Gynostemma pentaphyllum, J. Biol. Chem. 279, 41361-41367.

[16] G.L. Zhang, J.P. Deng, B.H. Wang, Z.W. Zhao, J. Li, L. Gao, B.L. Liu, J.R. Xong, X.D. Guo, Z.Q. Yan and G.D. Gao (2011). Gypenosides improve cognitive impairment induced by chronic cerebral hypoperfusion in rats by suppressing oxidative stress and astrocytic activation, Behav. Pharmacol. 22, 633-644.

[17] J. Yeo, Y.J. Kang, S.M. Jeon, U.J. Jung, M.K. Lee, H. Song and M.S. Choi (2008). Potential hypoglycemic effect of an ethanol extract of Gynostemma pentaphyllum in C57BL/KsJ-db/db mice, J. Med. Food 11, 709-716.

[18] M.C. Towler and D.G. Hardie (2007). AMP-activated protein kinase in metabolic control and insulin signalling, Circ. Res. 100, 328-341.

[19] Y. Shu, S.A. Sheardown, C. Brown, R.P. Owen, S. Zhang, R.A. Castro, A.G. Ianculescu, L. Yue, J.C. Lo, E.G. Burchard, C.M. Brett and K.M. Giacomini (2007). Effect of genetic variation in the organic cation transporter 1 (OCT1) on metformin action, J. Clin. Invest. 117, 1422-1431.

[20] D.G. Hardie (2011). AMP-activated protein kinase: an energy sensor that regulates all aspects of cell function, Genes Dev. 25, 1895-1908.

[21] D.G. Hardie, F.A. Ross and S.A. Hawley (2012). AMPK: a nutrient and energy sensor that maintains energy homeostasis, Nat. Rev. Mol. Cell Biol. 13, 251-262.

[22] D.G. Hardie and S.C. Lin (2017). AMP-activated protein kinase - not just an energy sensor, F1000Res. 6, 1724.

[23] L. Gong, S. Goswami, K.M. Giacomini, R.B. Altman and T.E. Klein (2012) Metformin pathways: pharmacokinetics and pharmacodynamics, Pharmacogenet. Genomics 22, 820-827.

[24] D.B. Shackelford and R.J. Shaw (2009). The LKB1-AMPK pathway: metabolism and growth control in tumour suppression, Nat. Rev. Cancer 9, 563-575.

[25] M. Kim, B.Y. Ahn, J.S. Lee, S.S. Chung, S. Lim, S.G. Park, H.S. Jung, H.K. Lee and K.S. Park (2009). The ginsenoside Rg3 has a stimulatory effect on insulin signaling in L6 myotubes, Biochem. Biophys. Res. Commun. 389, 70-73.

[26] T. Takemoto, S. Arihara, K. Yoshikawa, K. Kusumoto, I. Yano and T. Hayashi (1984). [Studies on the constituents of Cucurbitaceae plants. VI. On the saponin constituents of Luffa cylindrica Roem. (1)] Yakugaku, Zasshi 104, 246-255.

[27] S. Jager, C. Handschin, J. St-Pierre and B.M. Spiegelman (2007). AMP-activated protein kinase (AMPK) action in skeletal muscle via direct phosphorylation of PGC-1alpha, Proc. Natl. Acad. Sci. U. S. A. 104, 12017-12022.

[28] P.H. Thurairajah, W.K. Syn, D.A. Neil, D. Stell and G. Haydon (2005). Orlistat (Xenical)-induced subacute liver failure, Eur. J. Gastroenterol. Hepatol. 17, 1437-1438.

[29] J.W. Yun (2010). Possible anti-obesity therapeutics from nature--a review, Phytochemistry 71, $1625-1641$. 
Anti-obesity effects of Gynostemma pentaphyllum extract

[30] M. Wang, F. Wang, Y. Wang, X. Ma, M. Zhao and C. Zhao (2013). Metabonomics study of the therapeutic mechanism of Gynostemma pentaphyllum and atorvastatin for hyperlipidemia in rats, PLoS One 8 , e78731.

[31] D.E. James, L. Lederman and P.F. Pilch (1987). Purification of insulin-dependent exocytic vesicles containing the glucose transporter, J. Biol. Chem. 262, 11817-11824.

[32] S. Vilaro, M. Palacin, P.F. Pilch, X. Testar and A. Zorzano (1989). Expression of an insulin-regulatable glucose carrier in muscle and fat endothelial cells, Nature 342, 798-800.

[33] H.P. Lauritzen (2013). Insulin- and contraction-induced glucose transporter 4 traffic in muscle: insights from a novel imaging approach, Exerc. Sport Sci. Rev. 41, 77-86.

[34] P. Zhao, Q. Ming, M. Xiong, G. Song, L. Tan, D. Tian, J. Liu, Z. Huang, J. Ma, J. Shen, Q.H. Liu and X. Yang (2018). Dandelion chloroform extract promotes glucose uptake via the AMPK/GLUT4 pathway in L6 cells, Evid. Based. Complement Alternat. Med. 2018, 1709587.

[35] H.T.T. Pham, T.K.Q. Ha, H.M. Cho, B.W. Lee, J.P. An, V.O. Tran and W.K. Oh (2018). Insulin mimetic activity of 3,4- seco and hexanordammarane triterpenoids isolated from Gynostemma longipes, J. Nat. Prod. 81, 2470-2482.

[36] P. Puigserver, J. Rhee, J. Lin, Z. Wu, J.C. Yoon, C.Y. Zhang, S. Krauss, V.K. Mootha, B.B. Lowell and B.M. Spiegelman (2001). Cytokine stimulation of energy expenditure through p38 MAP kinase activation of PPARgamma coactivator-1, Mol. Cell 8, 971-982.

[37] D.C. Wright, P.C. Geiger, D.H. Han, T.E. Jones and J.O. Holloszy (2007). Calcium induces increases in peroxisome proliferator-activated receptor gamma coactivator-1alpha and mitochondrial biogenesis by a pathway leading to p38 mitogen-activated protein kinase activation, J. Biol. Chem. 282, 18793-18799.

[38] W.W. Winder, B.F. Holmes, D.S. Rubink, E.B. Jensen, M. Chen and J.O. Holloszy (2000). Activation of AMP-activated protein kinase increases mitochondrial enzymes in skeletal muscle, J. Appl. Physiol. 88, 2219-2226.

[39] X. Li, B. Monks, Q. Ge and M.J. Birnbaum (2007). Akt/PKB regulates hepatic metabolism by directly inhibiting PGC-1alpha transcription coactivator, Nature 447, 1012-1016.

[40] J.T. Rodgers, C. Lerin, Z. Gerhart-Hines and P. Puigserver (2008). Metabolic adaptations through the PGC-1 alpha and SIRT1 pathways, FEBS Lett. 582, 46-53.

[41] J.T. Rodgers, C. Lerin, W. Haas, S.P. Gygi, B.M. Spiegelman and P. Puigserver (2005). Nutrient control of glucose homeostasis through a complex of PGC-1alpha and SIRT1, Nature 434, 113-118.

[42] C. Canto, Z. Gerhart-Hines, J.N. Feige, M. Lagouge, L. Noriega, J.C. Milne, P.J. Elliott, P. Puigserver and J. Auwerx (2009). AMPK regulates energy expenditure by modulating NAD+ metabolism and SIRT1 activity, Nature 458, 1056-1060.

[43] N. Ferrara, B. Rinaldi, G. Corbi, V. Conti, P. Stiuso, S. Boccuti, G. Rengo, F. Rossi and A. Filippelli (2008). Exercise training promotes SIRT1 activity in aged rats, Rejuvenation Res. 11, 139-150.

[44] E.O. Ojuka (2004). Role of calcium and AMP kinase in the regulation of mitochondrial biogenesis and GLUT4 levels in muscle, Proc. Nutr. Soc. 63, 275-278.

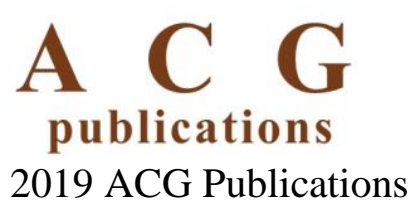

